\title{
KRULL DIMENSION AND REFLEXIVITY IN SOME NOETHERIAN RINGS
}

\author{
A. HAGHANY ${ }^{1}$ AND B. SARATH
}

\begin{abstract}
In this paper we study Noetherian prime rings $\boldsymbol{R}$ which satisfy the formula $|R|=\sup \left\{\left|I^{* *} / I\right|: I\right.$ is an essential left ideal of $\left.R\right\}+2$, where || denotes left Krull dimension. If further $Q / R$ is $|R|-1$ unmixed, where $Q$ is the simple Artinian quotient ring of $R$, we characterize $R$ using torsion theories cogenerated by the injective hulls of $|R|-1$ dimensional critical modules. Also equivalent statements are established, linking homological properties with dimension theory, for $R$-modules to be reflexive.
\end{abstract}

Introduction. Let $R$ be a left and right Noetherian ring. Then $R$ has left and right Krull dimension [1]. Denoting (left) Krull dimension by ||, it is well known that if further $R$ is prime, then $|R| \leqslant s(R)+2$ where $s(R)=\sup \left\{\left|I^{* *} / I\right|: I\right.$ is essential in $R$ \} and $I^{* *}=\operatorname{Hom}_{R}\left(\operatorname{Hom}_{R}(I, R), R\right)$. Commutative domains which satisfy $|R|=s(R)+2$ are classified in [6]. Some extensions to noncommutative rings are considered in $\S 1$. Adding the condition that $R=\cap R_{\tau}$ where $\tau$ ranges over torsion theories cogenerated by the injective hulls of $|R|-1$ dimensional critical modules, we characterize reflexive modules over $R$. In particular we show that in such rings an essential ideal $I$ of $R$ is reflexive if and only if the Krull dimension of any nonzero submodule of $R / I$ is $|R|-1$. In $\$ 3$ we consider prime rings $R$ satisfying $R=\cap R_{\tau}$ where each $R_{\tau}$ is a hereditary ring.

We would like to thank the referee for pointing out a serious error in an earlier version of this paper.

Notations. For an $R$-module $M$ we denote $\operatorname{Hom}_{R}(M, R)$ by $M^{*}$. Let $R$ be an order in a simple Artinian quotient ring $Q$ and let $I$ be a left $R$-submodule of $Q$ such that $I \cap R$ is essential. Then by $[6, I X, 5.1]$

$$
I^{*} \simeq I^{-1}=\{q \in Q \mid I q \subseteq R\} .
$$

A similar result holds for right submodules. Identifying $I^{*}$ with $I^{-1}$ we have $I \subseteq I^{* *}$ and $I^{*}=I^{* * *}$. Further $I$ is reflexive if and only if $I=I^{* *}$.

All rings have a 1 and all modules are unital. Unless otherwise specified, all properties are left properties. A regular ideal is a left ideal with a regular element. The Krull dimension is denoted by ||.

\footnotetext{
Received by the editors September 7, 1979 and, in revised form, June 10, 1980.

1980 Mathematics Subject Classification. Primary 16A08, 16A12, 16A33, 16A49, 16A55; Secondary 16 A48.

Key words and phrases. Reflexive module, Krull dimension, torsion theory.

'The author wishes to thank the Department of Pure Mathematics and Mathematical Statistics of the University of Cambridge for its warm hospitality during his sabbatical whilst part of the work was done. (c) 1981 American Mathematical Society $0002-9939 / 81 / 0000-0400 / \$ 02.75$
} 
For the rest of this paper $R$ is a prime left and right Noetherian ring. Denote the quotient ring of $R$ by $Q$ and $|R|$ by $\alpha$. We make the assumption that $\alpha$ is not a limit ordinal. A height one localisation $\tau$ over $R$ denotes localisation at a torsion theory cogenerated by the injective hull of a (nonzero) $\alpha-1$ dimensional cyclic critical $R$-module. Following [6] we write $R_{\tau}, M_{\tau}$ for the localisations of $R$ as a left module and arbitrary left $R$-modules $M$ respectively.

Definition 1.1. A ring $R$ has $(\mathrm{M} 2)$ if $\sup \left\{\left|I^{* *} / I\right|: I\right.$ is essential in $\left.R\right\}=\alpha-2$.

LEMMA 1.2. Let $M$ be an $R$-module with Krull dimension. Then $|M|<\alpha-2$ if and only if $M_{\tau}=0$ for every height one localisation $\tau$.

Proof. If $|M| \leqslant \alpha-2$ then $\operatorname{Hom}(M, E(A))=0$ for criticals $A$ of dimension $\alpha-1$ and hence $M_{\tau}=0$ for every $\tau$ of height one. Conversely if $|M|>\alpha-1$, there is an element $m$ in $M$ with $|R m|>\alpha-1$, and since $R m$ is Noetherian it has an $\alpha-1$ critical $A$ as a homomorphic image. If $\tau$ is cogenerated by $E(A)$ it is easily verified that $M_{\tau} \neq 0$.

LEMMA 1.3. Let $I$ be an ideal of $R$ and $\tau$ a height one localisation. If $I$ is $\tau$-torsion then $I$ is essential. Consequently $R_{\tau}=\left\{q \in Q: q \in J^{*}\right.$ for some $\tau$-torsion ideal $\left.J\right\}$.

Proof. Suppose there is a nonzero ideal $K$ in $R$ with $I \cap K=0$. Since $R$ is prime $K R$ contains a regular element. Hence $\operatorname{Hom}\left(K^{(n)}, E(A)\right) \neq 0$ for some $n$, and so $\operatorname{Hom}(K, E(A)) \neq 0$. It follows that $\operatorname{Hom}(R / I, E(A)) \neq 0$.

Proposition 1.4. Let $R$ have (M2) and suppose that $R_{\tau} I=I_{\tau}$ for every essential ideal $I$ of $R$ and every height one localisation $\tau$. Then every ideal of $R_{\tau}$ is reflexive.

Proof. From the exact sequence $0 \rightarrow I_{\tau} \rightarrow\left(I^{* *}\right)_{\tau} \rightarrow\left(I^{* *} / I\right)_{\tau}$ we get, using (1.2), that $I_{\tau}=\left(I^{* *}\right)_{\tau^{*}}$. Clearly $I^{*} R_{\tau} \subseteq\left(R_{\tau} I\right)^{*}$; hence $\left(I_{\tau}\right)^{* *} \subseteq\left(I^{*} R_{\tau}\right)^{*}$. Let $q \in\left(I^{* *}\right)_{\tau^{*}}$. Then there is a $\tau$-torsion ideal $J$ with $J q \subseteq I^{* *}$; hence $J q I^{*} \subseteq R$, that is, $q I^{*} \subseteq R_{\tau}$. Thus $q \in\left(I^{*} R_{\tau}\right)^{*}$. Since the argument is reversible we have $\left(I^{*} R_{\tau}\right)^{*}=\left(I^{* *}\right)_{\tau^{*}}$. Therefore $\left(I_{\tau}\right)^{* *} \subseteq\left(I^{* *}\right)_{\tau}=I_{\tau}$, and so $I_{\tau}$ is reflexive. Now if $T$ is an essential ideal of $R_{\tau}$, then $T \cap R$ is essential in $R$ and $R_{\tau}(T \cap R) \subseteq T \subseteq(T \cap R)_{\tau}$. Hence $T=(T \cap R)_{\tau}$ and by the above $T$ is reflexive.

LEMMA 1.5. Suppose that every ideal of $R_{\tau}$ is reflexive for every height one localisation $\tau$. Then $R_{\tau} I=I_{\tau}$ for every ideal $I$ of $R$.

Proof. It suffices to prove the lemma for essential ideals $I$. Clearly $\left(I_{\tau}\right)^{*} \subseteq$ $\left(R_{\tau} I\right)^{*}$. Let $q \in\left(R_{\tau} I\right)^{*}$. Then $q$ induces a homomorphism $I_{\tau} / R_{\tau} I \rightarrow Q / R_{\tau}$. Since $I_{\tau} / R_{\tau} I$ is $\tau$-torsion and $Q / R_{\tau}$ is $\tau$-torsion free $q$ must induce the zero map; hence $q \in\left(I_{\tau}\right)^{*}$. Thus $\left(I_{\tau}\right)^{*}=\left(R_{\tau} I\right)^{*}$, and so $\left(I_{\tau}\right)^{* *}=\left(R_{\tau} I\right)^{* *}$, that is $I_{\tau}=R_{\tau} I$.

THEOREM 1.6. Consider the following statements.

(i) $R$ has (M2) and $R_{\tau} I=I_{\tau}$ for every ideal $I$ of $R$ and every height one $\tau$.

(ii) Every ideal of $R_{\tau}$ is reflexive for every height one $\tau$.

Then (i) $\Rightarrow$ (ii). If each $R_{\tau}$ is left and right $R$-flat then (ii) $\Rightarrow$ (i). 
Proof. That (i) implies (ii) is by (1.4). Now suppose that each $R_{r}$ is flat on both sides and show that (ii) $\Rightarrow$ (i). From (1.5) it remains to prove that $R$ has (M2). Let $F \rightarrow G \rightarrow I \rightarrow 0$ be a free resolution of an essential ideal $I$ with $F$, $G$ finitely generated. There exists a commutative diagram

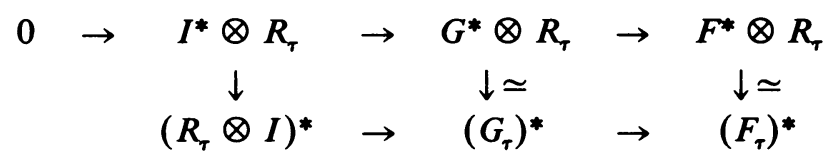

with exact rows since $R_{\tau}$ is flat. It follows that $I^{*} \otimes R_{\tau} \simeq\left(R_{\tau} \otimes I\right)^{*}$. Since $R_{\tau}$ is right $R$-flat $R_{\tau} \otimes I \simeq R_{\tau} I$; hence $I^{*} \otimes R_{\tau} \simeq\left(I_{\tau}\right)^{*}$. Thus $\left(I^{*} \otimes R_{\tau}\right)^{*} \simeq\left(I_{\tau}\right)^{* *}$ by (ii). Using $I^{*}=I^{* * *}$ we thus obtain $\left(I^{* *}\right)_{\tau}=\left(I_{\tau}\right)^{* *}=I_{\tau}$ and hence $R$ has (M2) by (1.2).

The next theorem shows that the conditions of (1.6) are equivalent for fully bounded classical prime rings. See [6, VII, 4.4].

Proposition 1.7. Let $\tau$ be a height one localisation, $C$ a subset of $R$ satisfying: an ideal $I$ is $\tau$-torsion if and only if $I \cap C \neq \varnothing$. Then any element of $C$ is regular and $R_{\tau}=C^{-1} R$. Consequently $R_{\tau}$ is right $R$-flat.

Proof. For $x$ in $C$ the ideal $R x$ is $\tau$-torsion and so, by (1.3), $x$ is regular. Hence $x$ is invertible in $Q$ and clearly $x^{-1}$ is in $R_{r}$. If $q \in R_{\tau}$ then $I q \subseteq R$ for some $\tau$-torsion ideal $I$. Hence there is an element $c$ in $I \cap C$ such that $q=c^{-1} r$ for some $r \in R$.

We need the following result which is immediate from $[4,2.3]$.

Proposition 1.8. Let $S$ be a left and right Noetherian left and right fully bounded ring. Let $M$ be an $S$-bimodule, finitely generated on both sides. Let $I, J$ be the left and right annihilators of $M$ respectively. Then $|S / I|=|M|=|S / J|$.

THEOREM 1.9. Let $R$ be $a$ left and right classical left and right fully bounded prime ring. Then $R_{\tau}$ is left and right $R$-flat for every height one localisation $\tau$. Consequently the conditiions of (1.6) are equivalent for $R$.

Proof. Let $\tau$ be a height one torsion theory cogenerated by $E(A)$, where $A$ is an $\alpha-1$ critical. Let $P=\operatorname{Ass}(A)$. Then $|R / P|=|A|$ and $\tau$ is also the torsion theory cogenerated by $E(R / P)$ [6, VII, §2]. Then $\operatorname{Hom}(R / I, E(A))=0$ implies that $\operatorname{Hom}(R / I+P, E(R / P))=0$ and this is possible only if $(I+P) / P$ is essential in $R / P$. Let $C(\tau)=\{c \in R:(R c+P) / P$ is essential in $R / P\}$. Then from the above, $I$ is $\tau$-torsion implies that $I \cap C(\tau) \neq \varnothing$. Conversely, suppose $R c+P / P$ is essential in $R / P$. Then since $R$ is fully bounded, $P \subseteq J \subseteq R c+P$ for some two-sided ideal $J$. Hence, if $I_{n}=\left(R c+P^{n+1}\right) \cap P^{n}$, we have $P^{n} J \subseteq I_{n}$, and hence that $\left|P^{n} / I_{n}\right| \leqslant\left|P^{n} / P^{n} J\right| \leqslant|R / J|$ by (1.8), and so $\left|P^{n} / I_{n}\right|<|R / J|<|R / P|=$ $|A|$. An induction now yields $\left|R /\left(R c+P^{n}\right)\right|<|R / P|$. If $c q=0$ for some $q \in$ $E(A)$ then since $P^{n} q=0$ for some $n$, we get $|R q|<|A|$ which yields $q=0$. Hence $\operatorname{Hom}(R / R c, E(A))=0$, that is $R c$ is $\tau$-torsion. Furthermore analogous arguments show that if $\sigma$ is the right torsion theory cogenerated by the injective hull of $R / P$ 
we get $T$ is $\sigma$-torsion if and only if $T \cap C(\sigma) \neq \varnothing$, where $C(\sigma)=\{c \in R$ : $(c R+P) / P$ is essential in $R / P\}$, and $T$ is a right ideal of $R$. But if $C=C(\sigma)=$ $C(\tau)$ then as in (1.7) it follows that $R_{\tau}=C^{-1} R=R C^{-1}$; hence $R_{\tau}$ is left and right $R$-flat.

REMARK 1.10. Theorem (1.6) can be modified for rings $R$ which are finitely generated as modules over a commutative Noetherian ring $K$ in the following way: " $R$ has (M2) $\Leftrightarrow$ every ideal of $R_{P}$ is reflexive". Here $P=\{x \in K \mid x A=0\}$ where $A$ ranges over $\alpha-1$ criticals, and $R_{P}$ denotes localisation at the multiplicative set $K-P$. ( $P$ is prime because $A$ is critical.) To prove this, it suffices to note that, by (1.8), $R$ has (M2) $\Leftrightarrow$ for every essential $I$ in $R$ and every $P$ as above, there is $x$ in $K-P$ with $x I^{* *} \subseteq I$. The result now follows easily. This leads us to ask the following question: "Can a classification theorem similar to (1.6) be obtained for fully bounded rings (or for all rings) by either modifying the localisations in a suitable way or by restricting them to only some height one localisations?"

\section{$\S 2$}

Definitions 2.1. (i) An $R$-module $M$ is said to be $\alpha-1$ unmixed if any nonzero finitely generated submodule of $M$ has Krull dimension $\alpha-1$.

(ii) We say $R$ has the height one intersection property if $R=\cap R_{\tau}$ where the intersection is taken over all height one localisations $\tau$.

LEMMA 2.2. $R$ has the height one intersection property if and only if $Q / R$ is $\alpha-1$ unmixed.

Proof. Let $0 \neq \bar{a} \in Q / R$. Suppose $|R a+R / R|=\alpha-1$. Then by (1.2) there exists a height one localisation $\tau$ such that $(R a+R / R)_{\tau} \neq 0$; hence $a \notin R_{\tau}$. Thus if $Q / R$ is $\alpha-1$ unmixed then $R=\cap R_{r}$. Conversely assume that $R$ has the height one intersection property. If $|R a+R / R|<\alpha-2$ then $R_{\tau} a \subseteq R_{\tau}$ for any $\tau$ of height one, and hence $a \in R$. It follows that $|R a+R / R|=\alpha-1$ and $Q / R$ is $\alpha-1$ unmixed.

LEMMA 2.3. Let $R$ have (M2) and the height one intersection property. Let $I \subseteq J$ be regular ideals. Then $I^{*}=J^{*}$ if and only if $|J / I| \leqslant \alpha-2$.

Proof. If $I^{*}=J^{*}$ then $J / I \subseteq I^{* *} / I$; hence $|J / I|<\alpha-2$ by (M2). To show the converse first note that $|R / L| \leqslant \alpha-2, L$ a regular ideal $\Rightarrow L^{*}=R$. For, if $q \in L^{*}-R$ we get a nonzero homomorphism $R / L \rightarrow Q / R$; hence $|R / L|=\alpha-$ 1 by (2.2). Now assume $|J / I| \leqslant \alpha-2$ and let $J=J_{0} \supseteq J_{1} \supseteq \cdots \supseteq J_{n}=I$ be a chain of ideals such that $J_{i} / J_{i+1} \simeq R / L_{i}$, for some ideals $L_{i}, i=0, \ldots, n-1$. Each $L_{i}$ is regular and $\left|R / L_{i}\right|<\alpha-2$. Hence $L_{i}^{*}=R$, which implies that $\operatorname{Ext}\left(R / L_{i}, R\right)=0$. Therefore $\operatorname{Ext}\left(J_{i} / J_{i+1}, R\right)=0$ for all $i$ and it follows that $\operatorname{Ext}(J / I, R)=0$, and hence $J^{*}=I^{*}$.

THEOREM 2.4. The following statements are equivalent.

(i) $R$ has (M2) and $Q / R$ is $\alpha-1$ unmixed.

(ii) $R$ has (M2) and the height one intersection property.

(iii) For any regular ideals $I \subseteq J, I^{*}=J^{*}$ if and only if $|J / I|<\alpha-2$. 
(iv) (a) $A$ proper regular ideal $I$ is reflexive if and only if $R / I$ is $\alpha-1$ unmixed;

(b) for regular ideals $I \subseteq J, I^{*}=J^{*}$ implies $\operatorname{Ext}(J / I, R)=0$;

(c) if $L$ is a regular ideal with $L^{*}=R$ then $|R / L|<\alpha-2$.

Proof. By (2.2) and (2.3) we have (i) $\Leftrightarrow$ (ii) $\Rightarrow$ (iii).

(iii) $\Rightarrow$ (iv). By assumption (c) holds, and to show (b) choose a chain of ideals $J=J_{0} \supseteq J_{1} \supseteq \cdots \supseteq J_{n}=I$ with $J_{i} / J_{i+1} \simeq R / L_{i}$ for $i=0, \ldots, n-1$. Then $I^{*}=J^{*} \Rightarrow|J / I| \leqslant \alpha-2$ by (iii). Hence $\left|R / L_{i}\right|<\alpha-2$, and so $L_{i}^{*}=R$ again by (iii). So $\operatorname{Ext}\left(R / L_{i}, R\right)=0$ and it follows that $\operatorname{Ext}(J / I, R)=0$. To see (a): let $I=I^{* *} \subset K$ such that $|K / I| \leqslant \alpha-2$. Then $I^{*}=K^{*}$, so $K=I$. Conversely if $R / I$ is $\alpha-1$ unmixed and $I \neq I^{* *}$ then $\left|I^{* *} / I\right|=\alpha-1$, which by (iii) gives $I^{*} \neq I^{* * *}$.

(iv) $\Rightarrow$ (i). Let $I$ be a regular ideal. Let $I \subseteq J \subseteq I^{* *}$ such that $J / I \simeq R / L$. Then $I^{*}=J^{*}$ and $L$ is a regular ideal. Hence $L^{*} / R \simeq \operatorname{Ext}(R / L, R) \simeq \operatorname{Ext}(J / I, R)=$ 0 , by (b). Thus by (c), $|R / L|<\alpha-2$. Therefore $|J / I|<\alpha-2$ and $R$ has (M2). If now $N$ is a finitely generated (nonzero) submodule of $Q / R$ then $N \simeq R / R c$ for some regular element $c$ in $R$. Hence $|R / R c|=\alpha-1$ by (a).

Next we classify finitely generated reflexive modules over rings which satisfy the equivalent conditions of (2.4).

THEOREM 2.5. Let $R$ satisfy the equivalent conditions of (2.4). Then the following statements are equivalent on a finitely generated $R$-module $M$.

(i) $M$ is reflexive.

(ii) There exists a finitely generated free module $F$ containing $M$ such that $\operatorname{Hom}(R / I, F / M)=0$ for any regular ideal I satisfying $I^{*}=R$.

(iii) There exists a finitely generated free module $F$ containing $M$ such that either $F=M$ or $F / M$ has no nonzero submodule of Krull dimension $<\alpha-2$.

(iv) $M$ is torsionless and $\operatorname{Ext}(R / I, M)=0$ for any regular ideal $I$ for which $I^{*}=R$.

Proof. (i) $\Rightarrow$ (ii). There exists an exact sequence $0 \rightarrow M \rightarrow F \rightarrow G$ with $F, G$ finitely generated free. Hence for any regular ideal $I$ (and in particular if $I^{*}=R$ ) we have $\operatorname{Hom}(R / I, F / M)=0$.

(ii) $\Leftrightarrow$ (iii). This is easily verified.

(ii) $\Rightarrow$ (iv). Certainly $M$ is torsionless and if $I$ is a regular ideal for which $I^{*}=R$ then the exact sequence $0 \rightarrow M \rightarrow F \rightarrow F / M \rightarrow 0$ yields

$$
\operatorname{Hom}(R / I, F / M) \rightarrow \operatorname{Ext}(R / I, M) \rightarrow \operatorname{Ext}(R / I, F)
$$

in which both end terms vanish.

(iv) $\Rightarrow$ (ii). This follows from the fact that since $M$ is torsionless there exists an exact sequence $0 \rightarrow M \rightarrow F \rightarrow F / M \rightarrow 0$ in which $F$ is finitely generated free.

(ii) $\Rightarrow$ (i). Suppose that $F$ as described in (ii) exists. We have an exact sequence $0 \rightarrow M \rightarrow M^{* *} \rightarrow N \rightarrow 0$, and we want to show that $N=0$. Since $R$ has (M2) it follows that $|N|<\alpha-2$. Suppose $N \neq 0$. Then there exists an ideal $I$ such that $|R / I| \leqslant \alpha-2$ and $\operatorname{Hom}(R / I, N) \neq 0$. Thus $I$ is regular with $I^{*}=R$. From the 
above sequence we obtain

$$
\operatorname{Hom}\left(R / I, M^{* *}\right) \rightarrow \operatorname{Hom}(R / I, N) \rightarrow \operatorname{Ext}(R / I, M)
$$

exact in which the first term vanishes. We obtain a contradiction by showing that $\operatorname{Ext}(R / I, M)=0$. Now the exact sequence $0 \rightarrow M \rightarrow F \rightarrow F / M \rightarrow 0$ yields $\operatorname{Hom}(R / I, F / M) \rightarrow \operatorname{Ext}(R / I, M) \rightarrow \operatorname{Ext}(R / I, F)$ exact. By (ii) the first term vanishes, and so does $\operatorname{Ext}(R / I, F)$ since $F$ is finitely generated free.

EXAMPLes 2.6. Among Noetherian prime rings satisfying the equivalent conditions of (2.4) are:

(i) Commutative Noetherian integrally closed domains $R$ with $0<|R|<\infty$.

(ii) Certain simple Ore extensions [4, 3.5].

(iii) Any flat maximal $K$-order in a central simple algebra over the quotient field of $K$, where $K$ is a commutative Noetherian integrally closed domain $[3,4.2]$.

$\$ 3$

In this section, we study rings $R$ that have the property that $R_{\tau}$ is hereditary for every height one localisation $\tau$. We need:

Definition 3.1. An element $x$ of $R$ is said to be critical if $R x$ is a critical $R$-module. When $x$ is critical, $c(x)$ denotes the sum of critical ideals of $R$ which have nonzero intersection with $R x$.

Lemma 3.2. Suppose $x \in R$ is critical and $x^{2} \neq 0$. Then

(i) $c(x)$ is critical.

(ii) $c(x) \cap l(x)=0$ where $l(x)$ is the left annihilator of $x$.

(iii) $l(x) \oplus R x$ and $l(x) \oplus c(x)$ are essential in $R$.

(iv) If $d$ is regular in $R$ and $a \in R$ then $R a \cap R a d \neq 0$.

Proof. Since $R$ is prime, $c(x)$ is nonsingular and (i) is clear. If $0 \neq c(x) \cap l(x)$, $\left|R x^{2}\right|<|R x|$. However $R x$ is critical; hence $x^{2}=0$, a contradiction. This shows (ii). Since $R / l(x) \simeq R x$ is critical and $R x \neq 0, l(x) \oplus R x$ is essential in $R$; hence (iii) follows. Finally for (iv), $R a \cap R a d=0$ implies that the sum $\Sigma_{i} \operatorname{Rad}^{i}$ is direct, a contradiction.

THEOREM 3.3. Let $R$ have (M2) and the height one intersection property. Suppose that all height one localisations over $R$ are hereditary. Let $x \in R$ be critical, $x^{2} \neq 0$. Then $R=c(x) \oplus l(x)$.

Proof. Let $\tau$ be of height one, $S=R_{\uparrow}$ and $L=$ left annihilator of $x$ in $S$. Since $S$ is hereditary and since projective ideals are reflexive, (1.5) shows that $S I=I_{\tau}$ for any ideal $I$ of $R$. For any ideal $K$ of $S$, since $K / K \cap R$ is $\tau$-torsion, $K=(K \cap R)_{\tau}$ $=S(K \cap R)$. Hence $L=S l(x)$ and the sequence

$$
0 \rightarrow L \rightarrow S \rightarrow S x \rightarrow 0
$$

is exact and splits, say by $x \rightarrow y$ for some $y$ in $S$. Then $y x=x$ and as $x, y$ have equal annihilators, $(1-y) x=0$ implies that $(1-y) y=0$, that is, $y=y^{2}$. Let $d=x+(y-1)$. Then $x=y x=y d \in S d$ and $y-1=(1-y) d \in S d$; hence $S d \supseteq S x+S(y-1)$. For any ideal $I$ of $R, S I / I$ is $\tau$-torsion; hence $I$ is essential in $S I$. Therefore by (3.2(iii)), $S x \cap L=0$ and $S x \oplus L$ is essential. But $L=$ $S(y-1)$, and $S d$ contains the essential ideal $S x \oplus L$; hence $d$ is regular in 
$S$. Since $y d=x$, it follows from (3.2(iv)) that $S x \cap S y \neq 0$. Let $I=S y \cap R$. Then $I \cap l(x)=0$ and $I \cap R x \neq 0$. Thus $I \subseteq c(x)$. Since $S I=S y$, we get $S(l(x) \oplus c(x))=S$. It follows from (1.2) that $|R / l(x) \oplus c(x)|<\alpha-2$, and hence from (2.3) that $l(x)^{* *} \oplus c(x)^{* *} \simeq R$. This isomorphism is identity on $l(x)$ and on $c(x)$. Therefore $R=T \oplus J$ with $T \supseteq l(x), J \supseteq c(x)$ and $|T / l(x)|<\alpha-2$. It follows that $T=l(x)$ and that $J$ is critical. From (3.1), $J=c(x)$ and the proof is complete.

Remark 3.4. Let $R$ be a Noetherian prime ring, $R \subseteq S_{\beta} \subseteq Q$ where the $S_{\beta}$ are hereditary rings which are $R$-flat. Then if $R=\cap S_{\beta}$, the conclusion of (3.3) is valid for $R$. Observe that if $S_{\beta} I=S_{\beta}$ for all $\beta$, then $I^{*} \subseteq S_{\beta}$ for all $\beta$; hence $I^{*}=R$. The rest of the proof of (3.3) holds unchanged.

\section{REFERENCES}

1. R. Gordon and J. C. Robson, Krull dimension, Mem. Amer. Math. Soc., no. 133, 1973.

2. A. Haghany, On duality and Krull dimension, J. London Math. Soc. (2) 14 (1976), 79-85.

3. __ Reflexive ideals in simple Ore extensions, J. London Math. Soc. (2) 16 (1977), 429-436.

4. A. V. Jategaonkar, Jacobsons conjecture and modules over fully bounded Noetherian rings, J. Algebra 30 (1974), 103-121.

5. R. Y. Sharp, A note on the dimensions of commutative Noetherian domains, J. London Math. Soc. (2) 115 (1977), 415-418.

6. B. Stenström, Rings of quotients, Springer-Verlag, Berlin, 1975.

Department of Mathematics, Shiraz University, Shiraz, Iran 